\title{
DISTRIBUTED HYBRID AGENT-BASED DISCRETE EVENT EMERGENCY MEDICAL SERVICES SIMULATION
}

\author{
Anastasia Anagnostou \\ Athar Nouman \\ Simon J.E. Taylor \\ Department of Information Systems, \\ Computing and Mathematics \\ Brunel University \\ Uxbridge, Middlesex UB8 3PH, UK
}

\begin{abstract}
This paper presents the development of a distributed hybrid agent-based (ABS) discrete event simulation (DES) model within the context of emergency medical services (EMS). The existing simulation models of EMS either are considered as a single model or several standalone models that represent different system elements in isolation. The aim of this research is to demonstrate the feasibility of using distributed simulation technology to implement hybrid EMS simulation. This would provide opportunities to study holistically integrated improvement scenarios for emergency medical services and crisis management systems. The case study is based on the London EMS and consists of an ambulance service ABS model and several accident and emergency departments DES models. Both the ABS and the DES models were developed in Repast Simphony toolkit using poRTIco RTI software to achieve communication between them. The results prove that we can use distributed simulation to successfully represent the real system.
\end{abstract}

\section{INTRODUCTION}

Discrete event simulation is being used to analyze problems in the healthcare sector for several decades(Brailsford et al. 2009). During which time, a growing number of healthcare providers worldwide have deployed simulation modeling in order to improve healthcare services. More recently, agent-based simulation has been adopted by operational research (OR) and is being used to model complex systems that consist of interacting agents (Heath et al. 2011). A significant proportion of this research has focused on EMS. For example, Ramirez et al. (2011) and Su and Shih (2003) use DES to model EMSs; Aringhieri (2010) employs combined ABS-DES approach for EMS analysis. EMSs are the units of the healthcare system that respond to medical emergencies and involve pre-hospital and/or in-hospital care. In this paper we focus on the two primary elements of an EMS: the ambulance service and the accident and emergency (A\&E) service.

As shown in Figure 1, the ambulance services can be either air- or land-based. Air ambulance services respond to serious injuries when timing is extremely critical. The land ambulance services coordinate the emergency calls and decide which vehicle and crew to be sent to an incident. Generally, the ambulance service fleet consist of fast response cars, ambulances and two-wheel vehicles. Furthermore, there are two types of crew: the basic life support crew that deal with non-life threatening incidents, and the advanced life support crew that are able to provide medical care and deal with life-threatening injuries. Depending on the incident, ambulances transfer patients to hospitals or just treat them at the scene.The second element of EMS are the A\&E departments. Patients arrive at A\&Eeither by ambulance or by their own means (walk-in). Ambulance arrivals are directed to the appropriate section (i.e., minors or majors) 


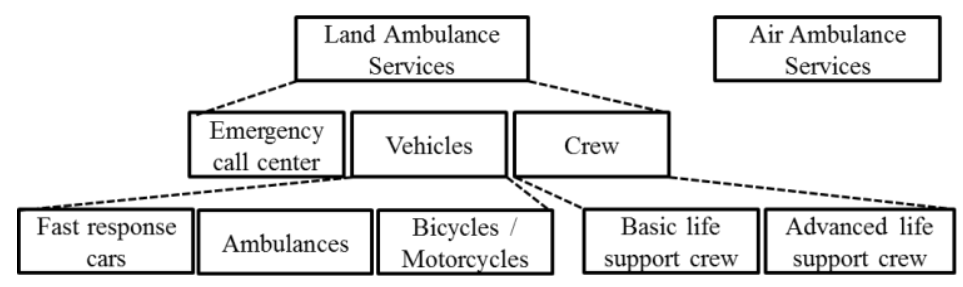

Figure 1: Ambulance Service Outline

according to their condition. Walk-in arrivals are directed to the above sections or leave the hospital after an initial assessment (i.e., triage service).

To develop a complete EMS model one would therefore need to simulate the models of each hospital and the ambulance service. This might involve the integration of these models into a single model. However, it might be argued that this is very inconvenient and potentially impossible if the models have been developed using different techniques and simulation packages. Distributed simulation (DS) provides an attractive alternative. DS perceives the models as independent simulation nodes where there is time synchronization and communication among them. The various simulations can run in different CPUs and communicate through a network or run in the same CPU.

As part of a larger research project, studying complex and large model integration, and the relationships between ABS-DES techniques, in this paper we present the development of a distributed hybrid simulation model. We are reporting the first findings of an ABS-DES hybrid model, where the London emergency medical services were used as the basis for the exemplar scenario. The first part of the model is an ABS simulation of the ambulance services and the second part is a DES simulation of various A\&E departments. The A\&E departments modelsare based on previous work (Eatock et al. 2011). The link between the two parts is achieved by distributed simulation technology and the data collection is based on online published data by the UK's department of health $(\mathrm{DoH})$ on the London ambulance and accident and emergency departments.

The aim of this paper is to demonstrate the feasibility of using a distributed ABS-DES simulation to model EMSs. The paper is structured as follows. In Section 2 there is a discussion on the EMS literature and on DS issues. Section 3 discusses in detail the conceptual design of the hybrid ABS-DES EMS model. Sections 4 and 5 present an analysis of the experimental design and a discussion of the results, respectively. Finally, Section 6 concludes the paper.

\section{BACKGROUND}

There are two main strands in this work: emergency medical service modeling and distributed simulation (supporting model reuse). We will discuss each of these in turn.

\subsection{Emergency Medical Service Modeling}

In simulation literature there are numerous studies of emergency medical services (EMS) (Barjis 2011). These studies focus on the improvement of EMSs, usually in an urban setting, on developing training tools, on methodological issues for providing better results and on crisis management and training. However, despite the large amount of simulation studies, they are usually conducted within the boundaries of an organization and in a one-time setting for supporting decision making when a major change is planned rather than supporting regular decision making. We now discuss the different approaches to EMS simulation from literature.

\subsubsection{Ambulance services models}

There are many existing models of ambulance services, most of them in the context of emergency services and disaster planning. Jotshi, Gong, and Batta (2009) study emergency vehicles dispatching and routing policies after an earthquake. Wu et al. (2008) use ABS, DES and geographical information sys- 
tems (GIS) to introduce a decision support system for real-time disaster management. Campbell and Schroder (2009) make use of simulation to present a training tool for emergency response. Henderson and Mason (2004) use DES to model the Auckland, New Zealand ambulance service and GIS for geospatial visualization. Their tool, BartSim, includes a travel model which produces deterministic computations of travel time when the travel time for the same distance varies during the day. DES is selected by Silva and Pinto (2010) to analyze ambulance services in a Brazil city. They use optimization techniques for ambulance deployment when testing different improvement scenarios.

DES is arguably not a natural choice for simulating ambulance services. ABS is a much more natural technique that can be used to model ambulance services. ABS is a simulation technique that is composed of autonomous agents and their environment. The agents have properties that evolve over time as they interact with each other and the environment. The agents are able to make decisions based on these properties. In an ambulance service model, the entities/agents are making decisions that depend on some properties and affect their behavior. For example, the ambulance crew will decide whether a patient needs onsite treatment or transfer to an A\&E. Also, if a route is blocked, an alternative route should be decided. EMSs in general and Ambulance services in particular are complex systems trying to balance political, economic and medical objectives (Henderson and Mason 2004). Traditional simulation techniques, such as DES, have not always managed to analyze adequately complex systems. According to North and Macal (2007), ABS will be the predominant simulation approach in the future for various, systems and technology related, reasons such as increasingly systems complexity, close similarity with object-oriented programming and increased computational power.

\subsubsection{Accident and emergency departments models}

Operations of the A\&E departments have received much attention in modeling and simulation (M\&S). Various simulation techniques have been deployed for A\&E analysis, typically using DES that seems to be the most popular technique in OR (Brailsford and Hilton 2001). Eatock at al. (2011) use DES to study the different A\&E strategies used to meet the four-hour length of stay operational standard imposed by the UK government. They reproduced accurately the different strategies used to meet the operational standards. DES is used by Komashie and Mousavi (2005) to analyze various key performance indicators in emergency departments. Alternatively, Escudero-Marin and Pidd (2011) employ ABS to model emergency departments, where the agents can change behavior according to some system variables.

\subsubsection{Emergency medical services models}

Ramirez et al. (2011) present a DES model of emergency care delivery systems. They use simulation optimization techniques to analyze ambulance diversion policies. They implement the model in a modular approach where, emergency incidents generation, ambulance decisions, and hospitals are separate modules. Su and Shih (2003) use simulation to evaluate and suggest improvements of EMSs. They use the DES technique to model the Taipei EMS. The coordination and synchronization of the basic and advanced life support units is done by an event controller object implemented in the eM-Plant simulation software. Their study focuses on EMS performance by testing different dispatch scenarios taking into account personnel utilization and waiting times. The Milan emergency service was modeled by Aringhieri (2010) using a combined ABS-DES approach. The model is built using AnyLogic simulation software which can combine ABS and DES. The service and travel times were modeled by introducing delays in a logistics-based DES model. By modeling the ambulance movements as delays, it is possible to assign a new mission to an ambulance in transit rather than when it is stationed. This employs the ABS approach where ambulances and operators are modeled as agents.

\subsection{Model Reuse and distributed simulation}

In this paper, we propose a framework that supports reusability of models. Reuse of simulation models has been debated among simulation experts and advantages and disadvantages of this practice have been 
discussed in the literature. In Robinson et al. (2004), reuse of models has been classified into three categories: basic modeling component reuse, reuse of subsystem models, and reuse of similar models. It is pointed out that careful recycling of simulation models has the potential to reduce modeling time and cost. Subsystem reusability issues, such as composability and data sharing problems, are discussed later in the paper. A key technology in achieving reuse is distributed simulation.

Distributed simulation can be defined as the distributed execution of a simulation program across multiple processors (Fujimoto 2000). In terms of this discussion, if we have several simulations running separately on several computers, then a distributed simulation is one in which these simulations cooperate (or interoperate) together, linked by a communications network and specialist software (middleware), as if they were a single simulation. Typically the software used to link simulations together is based on the IEEE 1516 High Level Architecture (HLA) [IEEE 2010]. This software is called a Run Time Infrastructure (RTI) and there are several presently available.

There are various motivations for using distributed simulation against attempting to reuse models by attempting to compose them in a single simulation package. Explored in full in Taylor et al. (2012), these include:

- Data transfer/access problems - a simulation often draws data from local data sources. As soon as a simulation leaves its domain of use the data must go with it. Data sources can be very large, multiple and/or connected to real-time sources. Moving these and ensuring that they are up to date may not be particularly convenient. This can lead to inaccuracies in results due to inconsistent data.

- Privacy - models and data may contain secrets that owners may not want to share openly.

- Model composability problems. Composing several models together in the same simulation software might not be as convenient as it sounds. Even if each of the stakeholders had previously developed models using the same commercial of-the-self simulation package (CSP), these models cannot simply be 'cut and pasted' into the same single model. Variable name clashes, global variables and different validation assumptions are three examples of the many problems of this approach. How do updates occur? How is version control maintained?

- Execution Time. As models grow in size, their process demands may increase predominately due to large event lists. Storage (RAM in particular) may also be strained by the larger model's demands.

Distributed simulation itself is not without its problems and can be extremely complex and often difficult to implement. However, it is attractive to think that the creation of large, distributed models that are private, access local up-to-date data, implement local changes efficiently and share the processing load of the model across the computers of the organizations is possible.

We now discuss the creation of our distributed EMS simulation.

\section{DESIGN}

\subsection{Conceptual framework}

The choice of the simulation technique is made based on two factors: the first is the appropriateness of the 
Anagnostou, Nouman, and Taylor

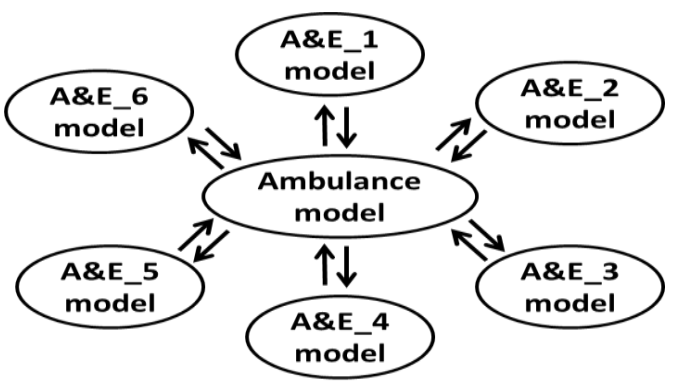

Figure 2: NosoPolis conceptual structure

method for each system and the second is our aim to produce a hybrid ABS-DES model. In our approach, the ABS and DES models are individual models that are linked together (interoperated) to form a hybrid EMS model. Communication between models is handled by a HLA RTI. There are two classes of models: the ambulance service model (ABS) and the A\&E models (DES). The conceptual structure of our system which we call NosoPolis is shown in Figure 2.

\subsection{NosoPolis ambulance service model}

ABS technique is selected for the ambulance service model due to high interaction of the simulation entities with each other and the environment. Simulation agents are of two types, passive agents that are part of the environment and active agents that interact with each other and the environment. Passive agents are the hospitals and the ambulance stations which have predetermined attributes such as location coordinates and capacity. The active agents are the emergency calls, the patients and the ambulances. Emergency calls are generated according to an arrival distribution. The generated emergency call carries location and incident information. Simultaneously with a call generation, a patient is generated that adapts the location and incident information from the call. Incident information is the patient condition and whether this patient needs transfer to an A\&E. In real ambulance services, when a call arrives at the dispatch center, an assessment is carried out and then the appropriate ambulance crew is sent to the incident scene. An assumption of our modelis that all calls will be attended, thus the emergency call is searching for the nearest available ambulance. Once an ambulance is found the call is removed from the simulation. When an ambulance is found, it is flagged as unavailable and starts the journey towards the incident scene. At the scene there is a delay for on-site treatment. If the patient is flagged as one that needs transfer to an A\&E, the ambulance searches for the nearest available A\&E and starts the journey to the hospital. After the patient's handover the ambulance goes back to station and is flagged as available. Travel times are calculated assuming an average ambulance speed and the Euclidean distance between the current location and the destination location. According to a study by Jones et al. (2010), Euclidean distance with a corrective factor can be used in research with a high degree of confidence to represent real driving distance in an urban setting. Silva and Pinto (2010) used a corrective coefficient to realistically represent the relationship between the actual distance and the Euclidean distance of two points in an urban environment. Each location point has an $\mathrm{X}$ and a $\mathrm{Y}$ coordinate, so the Euclidean distance $|\mathrm{s}|$ between two points is calculated by the following equation:

$$
\| s \mid=\sqrt{\left(X_{A}-X_{B}\right)^{2}+\left(Y_{A}-Y_{B}\right)^{2}}
$$

and the travel time is: $t=c * s / v$, where $t$ is the travel time in hours, $s$ is the distance in miles, $v$ is the average speed in miles per hour and $c$ is the corrective coefficient, which, in the case of London, is calculated to 1.32 . 


\subsection{NosoPolis accident and emergency model}

Discrete event simulation is selected as the technique for the accident and emergency department model due to the process oriented nature of A\&E departments. However it can be argued that emergency department entities are making decisions, our aim is not to model entities' behaviors but rather the processes within an A\&E.

We based this model on experiences from modeling A\&E departments in the UK (Eatock et al. 2011). In a general $A \& E$ model, there are two streams of patient input: the ambulance arrivals and the walk-in arrivals. Also, there are observation cubicles and ward beds for inpatients. In our prototype model, the A\&E service ends after the patients' observation. The inpatient ward of the A\&E is not modeled. Walk-in patients enter a queue for triage and then directed to the minors or majors department according to their condition. If no treatment is required, they leave the system after the triage service. Ambulance patients go directly to the minors or majors department, based on the previous communication with the ambulance model, and do not enter the subsequent queues. This is due to the fact that the hospital has been notified of the ambulance patient arrival and has reserved the appropriate resources. After the end of the service, patients exit the A\&E and the resources are released. Clinical staff is modeled as one type of resource, therefore there is no differentiation among nursing, medical or technical personnel. Also, another simplification of our model is that there is no imaging or lab tests process.

Table 1: Ambulance and A\&E models events

\begin{tabular}{|c|l|l|l|}
\hline \multicolumn{2}{|l|}{ Events of the ambulance model } & \multicolumn{2}{l|}{ Events of the A\&E model } \\
\hline$t_{\mathrm{a} 1}$ & Emergency call generation & $\mathrm{t}_{\mathrm{h} 1}$ & Patient arrival \\
\hline $\mathrm{t}_{\mathrm{a} 2}$ & An ambulance is found & $\mathrm{t}_{\mathrm{h} 2}$ & Triage queue entry \\
\hline $\mathrm{t}_{\mathrm{a} 3}$ & The selected ambulance arrived at the incident scene & $\mathrm{t}_{\mathrm{h} 3}$ & Triage service entry \\
\hline $\mathrm{t}_{\mathrm{a} 4}$ & An A\&E is found & $\mathrm{t}_{\mathrm{h} 4}$ & Minors queue entry \\
\hline $\mathrm{t}_{\mathrm{a} 5}$ & The ambulance departs from the incident scene & $\mathrm{t}_{\mathrm{h} 5}$ & Minors service entry \\
\hline$t_{\mathrm{a} 6}$ & The ambulance, carrying the patient, arrive at the A\&E & $\mathrm{t}_{\mathrm{h} 6}$ & Majors queue entry \\
\hline$t_{\mathrm{a} 7}$ & The ambulance arrive back to station & $\mathrm{t}_{\mathrm{h} 7}$ & Majors service entry \\
\hline & & $\mathrm{t}_{\mathrm{h} 8}$ & A\&E exit \\
\hline
\end{tabular}

The hospital availability is dependent on the clinical staff resources and the particular type of A\&E observation beds/cubicles (from now on are mentioned as beds), namely minors and majors. This can be calculated for each particular bed type as:

Hospital availability $=\min$ (available clinical staff, available beds), where

Available clinical staff $=$ clinical staff capacity-clinical staff occupancy

Available beds $s_{t y p e}=$ beds capacity type - beds occupancy type $_{\text {: }}$ type $=\{$ minors, majors $\}$

The hospital availability is updated in the A\&E model, this information is passed to the ambulance model in order to locate the suitable hospital when a patient transfer is needed.

The events of the ambulance service and A\&E models are shown in Table 1.

\subsection{Interoperability reference model}

A series of interoperability reference models (IRMs) have been developed and standardized to address model level interoperability issues between DES models [SISO-STD-006-2010] (SISO 2010). However these do not fully address model heterogeneity, when, for example, there are different simulation paradigms involved. To illustrate the interactions between the ambulance ABS and the A\&E DES models, the standardization of IRMs can be adopted and expanded. In this study, the interactions between the two parts of the hybrid model can be represented by a combined Type A.1 (entity transfer) \& Type C (shared event) IRM (Taylor et al. 2009). 


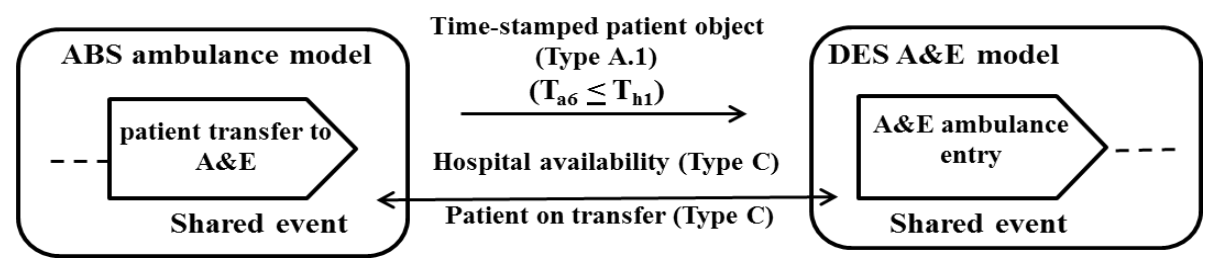

Figure 3: Type A.1 \& Type C Interoperability Reference Model

Figure 3 shows two model types that interoperate in the Nosopolis project.A detailed analysis of the interactions between the two models is given in Section 4. The Type A.1 aspect of the reference model indicates that when there is a need for hospital transfer then a patient object, or entity, passes from the ambulance model to the A\&E model. The time relationship should always be $T_{a b} \leq T_{h 1}$ (see Table 1). The Type $\mathrm{C}$ aspect of the reference model indicates that there are shared events between the two models such as the level of hospital availability and that a patient is on transfer. The ambulance notifies the A\&E when the decision for transfer is taken and not when the patient arrives at the hospital in order to avoid hospital availability conflict.

\subsection{Communication between the ambulance service and the A\&E models}

A typical distributed simulation is composed of number of simulation models which are known as federates and all these federates run under one federation connected through a typical RTI. In our case we have an ambulance model and six independent hospital models running as individual federates. The RTI provides information, synchronization, and coordination services between each federate. The diagram below (Figure 4) Illustrates how communication can take place between different models/federates implemented in Repast using standard RTI.

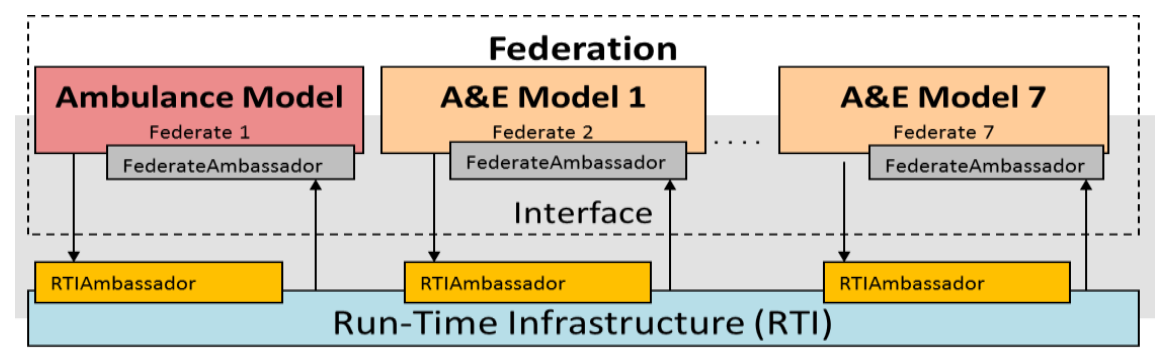

Figure 4: Emergency medical services federation

During the first time advance all hospital models update the ambulance model about their availability. The ambulance model always keeps an updated log of availability for its local A\&E, this information is send out by the hospital federates through RTI and get refreshed at every time tick if necessary. Once an ambulance has an emergency and needs to transfer a patient to an A\&E, it looks for the nearest hospital for the availability. The selection of the hospital is done on the basis of the distance from the patient's location and the availability of the hospital. Once a hospital is selected by the ambulance a notification is send to the particular hospital's A\&E to book a place through RTI. All communication among federates are time controlled by RTI (using Time Advance Request).

\section{IMPLEMENTATION AND EXPERIMENTAL DESIGN}

Both federate models were created in RePastSimphony (repast.sourceforge.net) toolkit. The HLA RTI interface was created using the poRTIco RTI software (www.porticoproject.org). Repast Simphony was selected for this project due to its scheduling and interoperability flexibility. Repast Simphony is a javabased simulation toolkit originally considered as an ABS tool but can be easily converted to a DES simulator. Both tools are open source and have been used in several simulation research projects (Kuhn et al. 


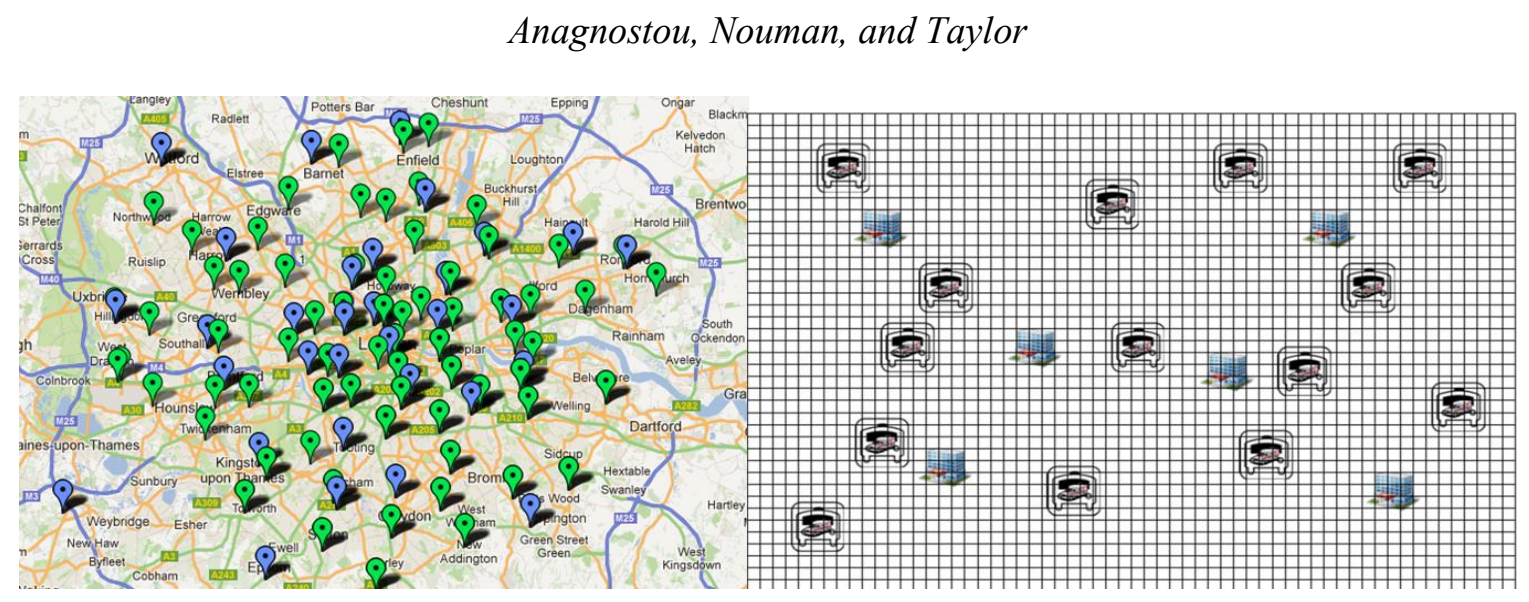

Figure 5: a) London ambulance and b) NosoPolis area

2010; Malik, Khan, and Hassan 2010; Minson and Theodoropoulos 2004; Tatara, Teymour, and Cinar 2007; Tu, Zacharewicz, and Chen 2011).

The ambulance model was constructed based on a simplified version of the London Ambulance Service (LAS). LAS covers an area of $620 \mathrm{mi}^{2}$ and has 70 ambulance stations and five Headquarters across Greater London. LAS has a fleet of 998 vehicles, 375 of which are ambulances (www.londonambulance.nhs.uk).

Greater London has 32 general A\&Es within the M25 ring (www.nhs.uk). As part of delivering the Government's reforms to the NHS, changes have been made in the A\&E departments across Greater London. In our data collection, we consider only the departments that were consistently part of the UK's Department of Health (DoH) data collection throughout 2011-12 (www.dh.gov.uk). The data has been aggregated and distributed normally to all A\&E hospitals across Greater London. In Figure 5a the blue landmarks indicate their location on the map and the green landmarks indicate the ambulance station locations. The average travel speed in London is generally low, ranging from less than $10 \mathrm{mph}$ in central London and up to $22 \mathrm{mph}$ in outer London (Steinbach et al. 2012). Therefore in the NosoPolis model the average speed is considered to be $15 \mathrm{mph}$.

The NosoPolismodel is an 1:5 scaling down emergency medical services system based on the London EMS.It can be seen from Figure 5a that in highly populated areas, i.e., central London, the density of available services is higher. In our model the call generation and the capacity of the A\&Es are equally distributed in the available space. Hence the ambulance stations and A\&Es are located in equally distributed distances (Figure 5b).

From the above analysis the specifications of our model are: cover area $=150 \mathrm{mi}^{2}$, number of A\&Es $=6$, number of ambulance stations $=14$, number of ambulances $=75$ and ambulance average speed $=$ $15 \mathrm{mph}$.

Although, the handover of a patient is one of the main reasons for ambulance delays and sometimes even increase the risk of adverse events (BBC news, Bost et al. 2012), at this stage of our study, we did not include it in our model. However it is planned to be incorporated in a later stage. Hence, referring to Table 1, the time relationship $t_{\mathrm{a} 6}=t_{\mathrm{h} 1}$ is always true, when the patient arrives at the hospital by ambulance.

The A\&E models are identical and their structure is a simplified version of a busy accident and emergency department in London. The different processes are aggregated. Therefore the department consist of three procedures (triage, minors and majors) in order to differentiate the main categorization of patients. For example, walk-in patients that need only advice exit the hospital straight after the triage service. Walk-in patients with minor injuries and more serious condition, after triage enter the minors and majors queue respectively. Patients that arrive by ambulance enter directly to minors or majors service without joining the queue. The total time of the services is the time that the patients spend with the clinical staff in the A\&E. The model configuration is based on literature and online data published by the UK NHS and the department of health. 
Anagnostou, Nouman, and Taylor

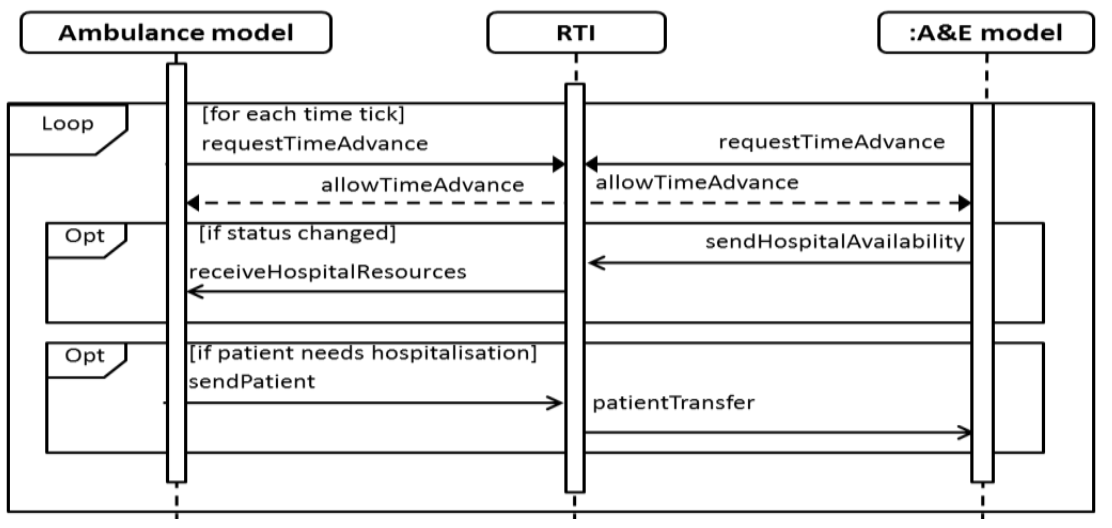

Figure 6: Interaction between the ambulance and each A\&E model

In each federate model there are two classes included for achieving communication with the RTI, an RTI ambassador class which is responsible for sending information to the RTI and a federate ambassador class which is responsible for receiving information from the RTI (Figure 4). A diagrammatic representation of the interactions between each federate and the RTI interface is shown in Figure 6. The ambulancemodel federate and each A\&E model federate send a time advance request to the RTI at every simulation timeunit (in Repast is called tick) and wait for the RTI response to allow time advance. When there is a change in the level of $A \& E$ availability, thisA\&E federate sends its availability to the ambulance federate. In a similar way, when the ambulance federate needs to transfer a patient to an A\&E it sends the patient instance to the selected A\&E federate. All A\&E federates have unique identifiers. Before any update is committed, the identifier of the A\&E that sends or receives the message is verified.

\section{RESULTS}

In the UK EMS there are several performance indicators that are set by the National Health Service (NHS). One crucial performance measurement for the ambulance service is the response time. That is the time from receiving the emergency call until the time that the ambulance arrives at the scene of incident. The target is that 75 per cent of category A calls (life threatening emergencies) are reached within eight minutes and 95 per cent within 19 minutes. From published data in the financial year 2011-12 LAS response time for 99 per cent of category A calls was $19 \mathrm{~min}$. The results from the NosoPolis model for one month simulation time are shown in Figure 7. As can be seen from the graph, the response time of 99 per cent of incidents was less than $19 \mathrm{~min}$.

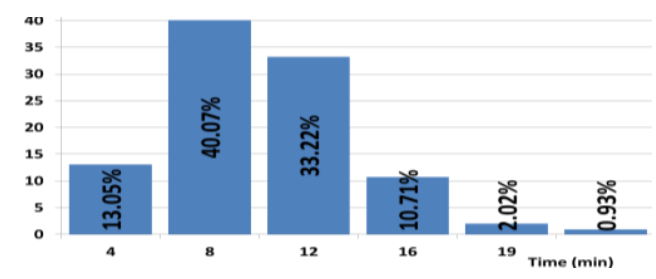

Figure 7: Ambulance service model response time

One key performance indicator for A\&E departments is the total duration of patient journey in the departments. The target for the UK's A\&E departments is to treat all patients within four hours. According to published data in the financial year 2011-12 for London strategic health authorities, 94 per cent of the attendances spent up to four hours in the A\&E departments. The duration in the NosoPolis's A\&E departments is shown in Figure 8. 


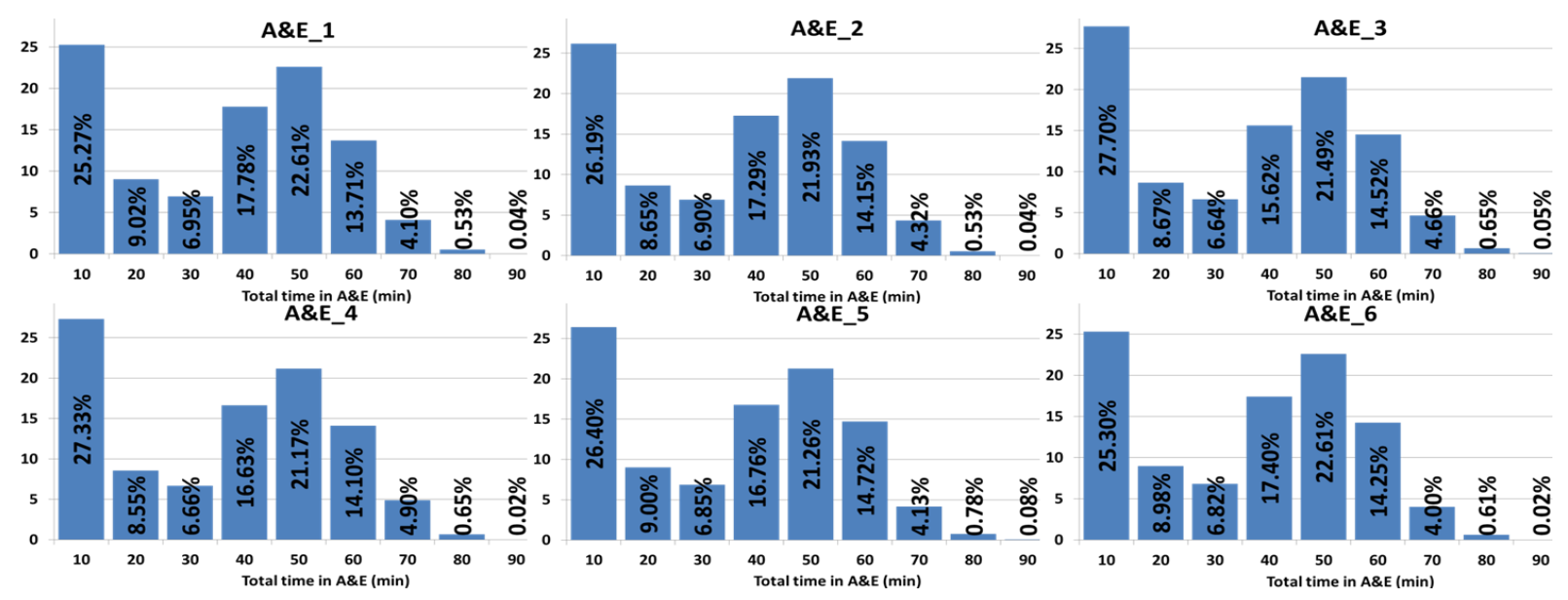

Figure 8: Total time spent in the six A\&E departments

This shows that the overall simulation behaves as expected according to its design. For example, the aggregation of all processes within the departments leads to less total time in an A\&E since there are not any queues in-between the various processes. Furthermore the fact that 40 per cent of the attendances need only advice (www.hsj.co.uk) leads to high volume of discharges in less than 30 minutes.

\section{CONCLUSIONS}

This paper presents a scaled down prototype model ofa distributed hybrid ABS-DES emergency medical services based on the London EMS. Since the aim of this research is to demonstrate the potentials of distributed hybrid simulation, the level of abstraction within the individual models is high. However, even with this low level of details, the results show a good approximation to the real system. Such a holistic system analysis tool can be a valuable resource for EMS management to explore improvement scenarios by performing "what-if" analysis. For example, we can study what effects will have on the system an increase in emergency calls arrivals. Another exploration area can be the ambulance deployment strategy.

This prototype model demonstrates the feasibility of using distributed simulation for a hybrid ABSDES EMS model. Also, it shows how an ABS-DES model can be realized and applied on other hybrid ABS-DES systems, i.e., modeling other than EMS exemplar scenarios. This technique enables the reuse of simulation models as subsystems without composing them in one large simulation. The use of a RTI middleware enables the various A\&E and the ambulance models to interoperate and behave as one system with independent components that communicate only when necessary.

The next steps of this project are to conduct performance testing of the distributed system. First, by increasing the level of details in the current model, that is for example, to disaggregate the A\&E processes such as investigation, clinical decision and treatment. Second, by increasing the scale of the system (A\&E departments and ambulance services) and reach the actual London EMS size.

\section{ACKNOWLEDGMENTS}

This research is funded by the Multidisciplinary Assessment of Technology Centre for Healthcare (MATCH), an Innovative Manufacturing Research Centre (IMRC) funded by the Engineering and Physical Sciences Research Council (EPSRC) (Ref: EP/F063822/1).

\section{REFERENCES}

Aringhieri, R. 2010. “An Integrated DE and AB Simulation Model for EMS Management.”IEEE Workshop on Healthcare Management, 1-6. Venice, Italy. 
Barjis J. 2011. "Healthcare Simulation and its Potential Areas and Future Trends."SCS M\&S Magazine 2:1-6.

BBC News. 2013. Accessed March 13. www.bbc.co.uk/news/uk-england-london-21039624

Bost, N., J. Crilly, E. Patterson, and W. Chaboyer. 2012. "Clinical handover of Patients Arriving by Ambulance to a Hospital Emergency Department: A Qualitative Study." International Emergency Nursing 20:133-141.

Brailsford, S., T. Bolt, C. Connell, J. H. Klein, and B. Patel. 2009. "Stakeholder Engagement in Health Care Simulation." In Proceedings of the 2009 Winter Simulation Conference, Edited by M. D. Rossetti, R. R. Hill, B. Johansson, A. Dunkin and R. G. Ingalls, 1840-1849. Austin, Texas.

Brailsford, S. C., and N. A. Hilton.2001. "A comparison of discrete event simulation and system dynamics for modelling healthcare systems." In Riley J (ed) Proceedings from ORAHS 2000, 18-39. Glasgow, Scotland.

Campbell, B. D. and K. E. Schroder. 2009. "Training for Emergency Response with RimSim: Response!” In Proceedings of the 2009 SPIE Defence, Security + Sensing Conference, Orlando, FL, USA.

Eatock, J., M. Clarke, C. Picton, and T. P. Young. 2011. "Meeting the Four-Hour Deadline in an A\&E Department." Journal of Health Organization and Management 25:606-624.

Escudero-Marin P., and M. Pidd. 2011. "Using ABMS to Simulate Emergency Departments." In Proceedings of the 2011 Winter Simulation Conference, Edited by S. Jain, R.R. Creasey, J. Himmelspach, K.P. White, and M. Fu, 1239-1250, Phoenix, AZ.

Fujimoto, R. M. 2000.Parallel and Distributed Simulation Systems. New York: John Wiley \& Sons.

Health Service Journal. 2011. Accessed March 13. http://www.hsj.co.uk/news/acute-care/emergency/40of-ae-patients-do-not-need-treatment/5024271.article

Heath, S. K., S. C. Brailsford, A. Buss, and C. M. Macal. 2011. "Cross-Paradigm Simulation Modeling: Challenges and Successes." In Proceedings of the 2011 Winter Simulation Conference, Edited by S. Jain, R. R. Creasey, J. Himmelspach, K. P. White, and M. Fu, 2788-2802, Phoenix, AZ.

Henderson, S. G. and A. J. Mason. 2004. "Ambulance Service Planning: Simulation and Data Visualization." In Operations Research and Health Care: A Handbook of Methods and Applications, Edited by M. L. Brandeau, F. Sainfort, and W.P. Pierskalla, 77-102. Boston: Kluwer Academic.

Jones, S. G., A. J. Ashby, S. R. Momin, and A. Naidoo. 2010. "Spatial Implications Associated with Using Euclidean Distance Measurements and Geographic Centroid Imputation in Health Care Research" Health Services Research 45(1):316-327.

Jotshi, A., Q. Gong, and R. Batta. 2009. "Dispatching and Routing of Emergency Vehicles in Disaster Mitigation Using Data Diffusion." Socio-Economic Planning Sciences 43:1-24.

Komashie, A., and A. Mousavi. 2005. "Modeling Emergency Departments Using Discrete Event Simulation Technique." In Proceedings of the 2005 Winter Simulation Conference, Edited by M. E. Kuhl, N. M. Steiger, F. B. Armstrong, and J. A. Joines, 2681-2685, Orlando, FL.

Kuhn,J. J .R. , J. F. Courtney, B. Morris, and E. R. Tatara. 2010."Agent-based analysis and simulation of the consumer airline market share for Frontier Airlines."Knowledge-Based Systems 23: 875-882.

Malik, A. W., S. A. Khan, and R. Hassan. 2010. "An HLA Based Real Time Simulation Engine for Manin-Loop Net Centric System," Journal of Engineering and Applied Sciences 7:47-54.

Minson, R., and G. K. Theodoropoulos. 2004. "Distributing RePast Agent-Based Simulations with HLA." Concurrency and Computation: Practice and Experience 20:1-25.

North, M. J., and C. M. Macal.2007, Managing Business Complexity. New York: Oxford University Press, Inc.

Ramirez, A., J. W. Fowler, and T. Wu. 2011. "Design of Centralized Ambulance Diversion Policies Using Simulation-Optimization." In Proceedings of the 2011 Winter Simulation Conference, Edited by S. Jain, R. R. Creasey, J. Himmelspach, K. P. White, and M. Fu, 1251-1262. Phoenix, AZ.

Robinson, S., R. E. Nance, R. J. Paul, M. Pidd, and S. J. E. Taylor. 2004. "Simulation Model Reuse: Definitions, Benefits and Obstacles." Simulation Modelling Practice and Theory 12:479-494. 
Silva, P. M. S., and L. R Pinto. 2010. "Emergency Medical Systems Analysis by Simulation and Optimization." In Proceedings of the 2010 Winter Simulation Conference, Edited by B. Johansson, S. Jain, J. Montoya-Torres, J. Hugan, and E. Yucesan, 2422-2432, Baltimore, MD.

SISO. 2010. SISO-STD-006-2010 Standard for COTS Simulation Package Interoperability Reference Models.Simulation Interoperability Standards Organization, Orlando, FL.

Steinbach R., J. Cairns, C. Grundy, and P. Edwards. 2012. "Cost benefit analysis of $20 \mathrm{mph}$ zones inLondon." Injury Prevention 00:1-3.

Su, S. and C. L. Shih. 2003. "Modeling an Emergency Medical Services System Using Computer Simulation.” International Journal of Medical Informatics 72:57-72.

Tatara, E., F. Teymour, and A. Cinar. 2007. "Control of Complex Distributed Systems with Distributed Intelligent Agents." Journal of Process Control 17:415-427.

Taylor, S. J. E., S. J. Turner, S. Strassburger, and N. Mustaffee. 2012, "Bridging The Gap: A StandardsBased Approach to OR/MS Distributed Simulation." ACM Transactions on Modeling and Computer Simulation 22(4): article 18.

Taylor, S. J. E., N. Mustafee, S. J. Turner, K. Pan, and S, Strassburger. 2009. "Commercial-Off-The-Shelf Simulation Package Interoperability: Issues and Futures." In Proceedings of the 2009 Winter Simulation Conference, Edited by M. D. Rossetti, R. R. Hill, B. Johansson, A. Dunkin and R. G. Ingalls, 203-215. Austin, Texas.

The Department of Health. 2013. Accessed March 2013. www.dh.gov.uk.

The portico Project. 2013. Accessed 2013. www.porticoproject.org.

The Repast Suite. 2013. Accessed March 13. repast.sourceforge.net.

Tu, Z., G. Zacharewicz, and D. Chen. 2011. "Developing a Web-Enabled Federate Based on poRTIco RTI.” In Proceedings of the 2011 Winter Simulation Conference, Edited by S. Jain, R. R. Creasey, J. Himmelspach, K. P. White, and M. Fu, 2289-2301, Phoenix, AZ.

Wu, S., L. Shuman, B. Bidanda, O. Prokopyev, M. Kelley, K. Sochats, and C. Balaban. 2008. "Simulation-based Decision Support System for Real-time Disaster Response Management." In Proceedings of the 2008 Industrial Engineering Research Conference, Edited by J. Fowler, and S. Mason, 58-63. Vancouver, Canada.

\section{AUTHOR BIOGRAPHIES}

ANASTASIA ANAGNOSTOU is a PhD candidate at School of Information Systems, Computing and Mathematics, Brunel University, UK. She holds an MSc in Telemedicine and e-Health Systems and a BSc in Electronic Engineering. Her research interests are related to the application of modelling and simulation techniques within the healthcare sector. Her email address is Anastasia.Anagnostou01@brunel.ac.uk.

ATHAR NOUMAN is a PhD candidate at School of Information Systems, Computing and Mathematics, Brunel University, UK. He holds an MSc in Distributed Systems and an MSc in Software Engineering and a BSc in Computer Science. His research interests are related to distributed modeling and simulation and high performance computing. His email address is Athar.Nouman@brunel.ac.uk.

SIMON J.E.TAYLOR is the Founder and Chair of the CSPI PDG under SISO. He is the co-founding Editor-in-Chief of the UK Operational Research Society's (ORS) Journal of Simulation and the Simulation Workshop series. He was Chair of ACM's SIGSIM (2005-2008). He is a Reader in the School of Information Systems, Computing and Mathematics at Brunel and leads the ICT Innovation Group. He has published over 150 articles in modeling and simulation. His recent work has focused on the development of standards for distributed simulation in industry. His email address is Simon.Taylor@brunel.ac.uk. 